\title{
Intraoperative vs 24-Hour Administration of Cefamandole to Prevent Deep Sternal Wound Infection and Endocarditis after Adult Cardiac Surgery
}

\author{
Jean-Michel Maillet $^{*}$, Stéphane Thierry ${ }^{1}$, Grégoire Oghina² ${ }^{2}$ Paul Le Besnerais ${ }^{3}$, Patrick Mesnildrey ${ }^{3}$, \\ Nicolas Bonnet ${ }^{3}$, François Simoneau ${ }^{4}$, Denis Brodaty ${ }^{1}$ \\ ${ }^{1}$ Department of Cardiovascular and Thoracic Intensive Care, Centre Cardiologique du Nord, Saint-Denis, France; ${ }^{2}$ Department of \\ Microbiology, Centre Cardiologique du Nord, Saint-Denis, France; ${ }^{3}$ Department of Cardiac Surgery, Centre Cardiologique du Nord, \\ Saint-Denis, France; ${ }^{4}$ Department of Anesthesiology, Centre Cardiologique du Nord, Saint-Denis, France. \\ Email: ${ }^{*}$ jm.maillet@ccncardio.com
}

Received September $6^{\text {th }}, 2013$; revised September $26^{\text {th }}, 2013$; accepted October $6^{\text {th }}, 2013$

Copyright (C) 2013 Jean-Michel Maillet et al. This is an open access article distributed under the Creative Commons Attribution License, which permits unrestricted use, distribution, and reproduction in any medium, provided the original work is properly cited.

\begin{abstract}
Background and Objectives: Duration of antibiotic prophylaxis for cardiac surgery is still debated and controversial. International guidelines are vague: French guidelines recommend an intraoperative administration, while the Society of Thoracic Surgeons' guidelines suggest that optimal postoperative prophylactic antibiotics be given for 48 hours or less. Very few studies have compared the same antibiotic with 2 different administration durations. The study was designed to compare the efficacy of 24-hour administration of cefamandole vs intraoperative cefamandole to prevent deep sternal wound infection and endocarditis after cardiac surgery. Methods: This retrospective and observational study compared the rates of severe surgical site infections (deep sternal wound infection, endocarditis) after cardiac surgery between period 1, 01/01/2008-31/08/2008, with 24-hour administration of cefamandole, and period 2, 01/09/2008-30/04/2009 with intraoperative cefamandole. Results: Among 933 patients, 14 patients $(1.5 \%)$ developed surgical site infection during the 16-month study: $1.3 \%$ during the first period and $1.7 \%$ during the second (ns). The populations (470 patients in period 1 and 463 in period 2) were homogeneous and comparable for pre-, intra- and postoperative characteristics. Surgical site infection characteristics (pathogens involved, time to diagnosis) and consequences (longer hospital stay, outcomes) were comparable in the 2 groups. Conclusions: Intraoperative cefamandole was as safe as its 24-hour administration to prevent deep sternal wound infection and endocarditis after adult cardiac surgery.
\end{abstract}

Keywords: Surgical Site Infection; Deep Sternal Wound Infection; Endocarditis; Antibiotic Prophylaxis; Cardiac Surgery; Cefamandole

\section{Introduction}

"Does the duration of perioperative antibiotic prophylaxis matter in cardiac surgery?" was the provocative title of a systematic review and meta-analysis recently published by Mertz and associates [1]. Shorter duration of antibiotic prophylaxis definitively has many advantages: lower costs, decrease selection pathogen pressure [2], less drug toxicity and simplification of the very complex procedure of correct routine daily administration of antibiotic prophylaxis [3]. Duration of antibiotic prophylaxis remains controversial. The Society of Thoracic Sur-

${ }^{*}$ Corresponding author. geons' recommendations are vague, seem to support a duration of $\geq 24$ hours, perhaps less, but no longer than $\geq 48$ hours [4]. Mertz and associates' conclusions were elusive because of the lack of well-designed studies and emphasized the heterogeneity of antibiotic regimens and the risk of bias in the published studies [1]. In our institute, the local Nosocomial Infection Control Committee modified and shortened the antibiotic prophylaxis duration with single dose despite some local surgeons' reluctance. This study was undertaken to evaluate whether intraoperative cefamandole was as effective as its 24hour administration to prevent the most severe SSI after adult cardiac surgery, deep sternal wound infection (DSWI) and endocarditis. 


\section{Methods}

During a 16-month period, we retrospectively studied all consecutive adults ( $>18$ years of age) who underwent cardiac surgery with cardiopulmonary bypass (CPB): period 1: $01 / 01 / 2008$ to $31 / 08 / 2008$ and period 2: $01 / 09 /$ 2008 to $30 / 04 / 2009$. No included in this study were patients with $\beta$-lactam allergy, acute endocarditis and/or vascular surgery with CPB. Our institution is private with more than 200 beds dedicated exclusively to medical and surgical cardiovascular and thoracic pathologies and where $700-850$ interventions are performed annually.

The following parameters were extracted from the local database which is registred at the French National Commission Informatics and Liberty: sex, age, body mass index, cardiovascular risk factors (diabetes, current smoker, hypercholesterolemia, hypertension), presence of chronic obstructive pulmonary disease (COPD), chronic renal insufficiency (creatinemia $\geq 200 \mu \mathrm{mol} / \mathrm{L}$ ), peripheral vascular disease, type of cardiac surgery [coronary artery bypass graft $(\mathrm{CABG}) \pm$ valve replacement (VR), VR and other], use of 1 or 2 internal thoracic arteries, Euroscore I [5], duration of ICU length of stay, prolonged mechanical ventilation ( $\geq 48$ hours), use of noninvasive ventilation, red-cell transfusion, vasopressor use, rate of acute renal insufficiency (defined as a 2-fold increase of the preoperative creatinemia), dialysis required.

In our institution, anesthesiologists and intensivists are responsible for antibiotic-prophylaxis prescription, intraoperatively and post-operatively, respectively. Each prescriber received the written local protocol recommendation before the switch to the shorter regimen in August 2008. This local protocol, validated by our local Nosocomial Infection-Control Committee, recommends the use of cefamandole as the first-line antibiotic in the absence of $\beta$-lactam allergy. In the operating room, infusion of $1.5 \mathrm{~g}$ of cefamandole within the 1 hour preceding the incision is recommended. It is also specified that another infusion $750 \mathrm{mg}$ of cefamandole is mandatory when the surgery lasts $\geq 120$ minutes, with repeated infusions every 120 minutes, as needed. Lastly, for period $1,750 \mathrm{mg}$ of cefamandole were infused every 6 hours during the first 24 postoperative hours during the intensive care unit stay.

The Centers for Disease Control definitions were used to for DSWI and endocarditis [6]. Occurrence of those infections was recorded and validated independently by our local Nosocomial Infection-Control Committee. For each infection, we studied the date of its diagnosis, the interval between surgery and infection, the pathogen(s) involved, their susceptibilities to antibiotics and the development of concomitant bacteriemia. All patients with healing problems, suspected wound infection or suspected postoperative endocarditis were systematically re- addressed to their surgeon postoperatively.

There were no changes in measures to prevent infection between the study periods. Skin preparation was performed as follow: the night before surgery, patients took two showers with an antiseptic solution. The day of surgery, 2 hours before surgery, patients took a third shower with an antiseptic solution and hair was removed. No specific outbreaks occurred during the study period.

The concerned database had been approved by the French national commission for computerized files and liberty. According to French law, its subsequent use for epidemiologic work does not require additional regulatory or ethic commission approval.

\section{Statistical Analysis}

Continuous variables are presented as median with range and categorical variables as $\mathrm{n}(\%)$. Comparisons were made using the chi-square or Fisher's exact test (when needed) to analyze differences in categorical variables. The Student's t-test or the Mann-Witney test was used, as appropriate, for continuous variables. A $p$ value $<0.05$ was considered statistically significant. Statistical analyses were performed using Statview version 5.1 software (SAS Institute Inc., Cary, NC).

\section{Results}

During the study, 987 adults [499 (50.5\%) during period 1 and 488 (49.5\%) during period 2] had heart surgery with CPB in our institution. Fifty-four patients were not included, 29 from period 1 and 25 from period 2, because of $\beta$-lactam allergy, acute endocarditis and/or vascular surgery with CPB. Thus, 933 patients were included in the study: 470 during period 1 and 463 during period 2.

Detailed preoperative, intraoperative and postoperative patient characteristics, categorized according to the antibiotic regimen used during those periods, are given in Table 1. The 2 groups were homogeneous and comparable for their demographic profiles and clinical characteristics. The 3 surgeons' activities were comparable during the 2 periods (data not shown).

During the study, 14 (1.5\%) SSI were diagnosed: $1.3 \%$ $(\mathrm{n}=6)$ during period 1 vs $1.7 \%(\mathrm{n}=8)$ during for period 2. SSI characteristics were comparable concerning the median time to infection onset 28 days (range, 5 - 129 days) vs. 23 days (range, 4 - 112 days). Eleven (5 in period 1 and 6 in period 2) bacteriemia were diagnosed with SSI. Of 17 pathogens isolated, $12(70.5 \%)$ were Gram-positive cocci and 5 (20.9\%) Gram-negative bacilli. They were similarly distributed in the 2 periods.

Median postoperative hospital stay was comparable during periods: 1 and 2, respectively: 9 (range 0 - 372) vs 9 (range 0 - 113) days. SSI occurrence significantly pro- 
Table 1. Preoperative, intraoperative and postoperative characteristics.

\begin{tabular}{|c|c|c|c|}
\hline & Period $1 n=470$ & Period $2 n=463$ & $p$-Value \\
\hline \multicolumn{4}{|l|}{ Preoperative characteristic } \\
\hline Sex, male $(\%)$ & $331(70.4)$ & $352(76.0)$ & 0.94 \\
\hline Age (years), median (range) & $69(19-96)$ & $68(18-89)$ & 0.39 \\
\hline Body Mass Index $\left(\mathrm{kg} / \mathrm{m}^{2}\right)$, median (range) & $27(17-53)$ & $27(14-44)$ & 0.44 \\
\hline Hypertension, (\%) & $265(56.4)$ & $267(57.7)$ & 0.74 \\
\hline Diabetes, $(\%)$ & $130(27.7)$ & $137(29.6)$ & 0.51 \\
\hline Hypercholesterolemia, (\%) & $235(50)$ & $227(49.0)$ & 0.74 \\
\hline Current smoker, (\%) & $78(16.6)$ & $63(13.6)$ & 0.23 \\
\hline Euroscore, point, median (range) & $5(0-18)$ & $5(0-16)$ & 0.69 \\
\hline \multicolumn{4}{|l|}{ Intraoperative characteristic } \\
\hline Type of surgery, (\%) & & & 0.37 \\
\hline $\mathrm{CABG} \pm \mathrm{VR}$ & $264(56.2)$ & $278(60.0)$ & \\
\hline VR & $162(34.5)$ & $142(30.7)$ & \\
\hline Others & $44(9.4)$ & $43(9.3)$ & \\
\hline Total CPB time (min), median (range) & $73(29-287)$ & $75(14-251)$ & 0.4 \\
\hline Aortic cross clamp time (min), median (range) & $55(0-205)$ & $55(8-203)$ & 0.59 \\
\hline Bilateral ITA $(n=264$ period $1 ; n=278$ period 2$)$ & $220(83.3)$ & $236(84.9)$ & 0.68 \\
\hline \multicolumn{4}{|l|}{ Postoperative course } \\
\hline ICU LOS (days), median (range) & $2(1-50)$ & $2(0-56)$ & 0.89 \\
\hline $\mathrm{MV} \geq 2$ days, median (range) & $31(6.6)$ & $39(8.9)$ & 0.32 \\
\hline Vasopressor use (\%) & $181(38.5)$ & $171(36.9)$ & 0.63 \\
\hline Noninvasive ventilation (\%) & $41(8.7)$ & $40(8.6)$ & 0.96 \\
\hline Acute renal insufficiency (\%) & $34(7.2)$ & $41(8.9)$ & 0.4 \\
\hline Hemodialysis (\%) & $16(3.4)$ & $17(3.7)$ & 0.86 \\
\hline Red-cell transfusion (\%) & $163(34.7)$ & $155(33.5)$ & 0.73 \\
\hline Postoperative hospital LOS (days), median (range) & $9(0-372)$ & $9(0-113)$ & 0.82 \\
\hline Hospital mortality (\%) & $28(6)$ & $34(7.3)$ & 0.43 \\
\hline
\end{tabular}

Data are presented as mediane, range and \%. CABG: coronary artery-bypass graft; CPB: cardiopulmonary bypass; ICU: intensive care unit; ITA: internal thoracic artery; LOS: length of stay; MV: mechanical ventilation; VR: valve replacement.

longed the median postoperative hospital stay from 9 (range 0 - 372) days without to 30 (range 5 - 77) days with SSI $(p<0.0002)$, and comparably during the 2 periods. Mortality rates were comparable for periods 1 and 2, respectively, $6 \%$ and $7.3 \%$. Mortality rate for severe SSI was $35.7 \%$ for the study period.

\section{Discussion}

The results of this retrospective study showed that intra- operative cefamandole prophylaxis was as effective as 24-hour administration to prevent the most severe surgical site infections after adult cardiac surgery, DSWI and endocarditis.

Antibiotic prophylaxis is one of the most important preventive measures against SSI after major surgery, particularly, cardiac surgery [4]. Because of the high morbidity and mortality associated with DSWI and endocarditis, an adequate antibiotic regimen and its ad- 
ministration are essential. The duration of antibiotic prophylaxis is still being debated and remains controversial. Recently the Society of Thoracic Surgeons' guidelines underscored the heterogeneity of studies (eg recommendations from different scientific societies concerning this specific question) and recommended that postoperative optimal prophylactic antibiotics (class IIa, level B) be given $\leq 48$ hours [4]. In 2010, French guidelines validated a very firm position recommending the shorter administration for cardiac surgery with only intraoperative administration of prophylactic cefamandole or cefazolin [7]. A recent systematic review and meta-analysis showed that perioperative antibiotic prophylaxis lasting $\geq 24$ hours might be more effective than shorter regimen but the findings were limited by the heterogeneity of antibiotic regimens and the risk of bias in the published studies [1]. Some studies compared single-dose vs longer antibiotic prophylaxis [1], but very few used the same antibiotic in both arms [8,9] and only the former compared singledose vs 24-hour doses of the same antibiotic. Tamayo and associates, in their monocentric unblinded prospective study, compared 2 cefazolin doses over 42 months: the single-dose was associated with significantly higher SSI rate $(8.3 \%)$ than with multiple-doses $(3.6 \%)(p=$ $0.004)$. That significant difference was due to the significantly higher single dose SSWI rate $(5 \%)$ than with multiple doses $(1.7 \%)(p=0.007)$. Notably, DSWI and endocarditis rates were comparable for both groups (respectively $1.9 \%$ and $1.2 \%$ for single dose vs. $1.2 \%$ and $0.2 \%$ with 24 -h regimen) comparable to those of current study [9].

Achieving rigorous daily antibiotic prophylaxis regimen is very difficult [3]. The antibiotic-prophylaxis protocol has many steps that must be respected. At each step, non-adherence is possible; the type of antibiotic, the timing of its prophylactic administration, the dose, the need for repeated infusions because of prolonged surgery and postoperative duration [3]. Single-dose has the major advantage of simplifying the antibiotic-prophylaxis protocol.

Cefamandole is a second-generation cephalosporin recommended as antibiotic prophylaxis in first line in cardiac surgery in France [7], because it is safe, inexpensive, bactericidal against Gram-positive cocci (MSSA and MSCNS) and most Gram-negative bacilli and has a good diffusion. Repeated administrations for surgery lasting $>2$ hours are required because of its short half-life. It was effective against $>85 \%$ of the pathogens involved in the SSI.

Antibiotic prophylaxis is the one of the most effective preventive measures against SSI. However, the SSI rate after cardiac surgery range from $0.25 \%$ to $4 \%$ for DSWI [4]; those values remain high, particularly for this type of surgery classified as "clean surgery". Many factors explain that fact (eg, older age patient, more co-morbidities and/or generalization of bilateral use of internal thoracic arteries). In this context, new strategies have been developed to try to lower the SSI rate: preoperative mupirocin, collagen-gentamicin implants and triclosan-coated sutures. Hopes raised by these approaches have not yet been fulfilled [10-12]. It seems that the research concerning antibiotic prophylaxis has been left by the wayside, despite the numerous unanswered questions. Only, an international multicenter study will be able to answer the following question: What is the best duration of antibiotic prophylaxis? However, institutional and/or pharmaceutical financial support is lacking, even though millions of euros or dollars spent on useless antibiotic administration could be saved if single-dose antibiotic prophylaxis were to be demonstrated as safe as 24 -hour or 48-hour administration.

The major limitation of our study is its retrospective design. However, the 2 populations, with $>400$ patients in each arm, were comparable concerning important pre-, intra- and postoperative factors associated with SSI thereby rendering possible interpretation of our results. We tried to be as closed as possible to the "real world" including patients who underwent emergency surgery and limiting the exclusion criteria. In addition, the short duration of the study (16 months) limited the bias related to variations of daily patients care. Second, the sample is small. Lastly, because of the retrospective nature of the study, no power calculation is allowed methodologically and was not performed, and a type 2 error could be possible.

\section{Conclusion}

Our results suggest that single-intraoperative dose cefamandole is as effective as its 24-hour administration to prevent the most severe SSI after adult cardiac surgery. Well-designed prospective studies are urgently needed to resolve this controversial and important issue.

\section{REFERENCES}

[1] D. Mertz, J. Johnstone and M. Loeb, "Does Duration of Perioperative Antibiotic Prophylaxis Matter in Cardiac Surgery? A Systematic Review and Meta-Analysis," Annals of Surgery, Vol. 254, No. 1, 2011, pp. 48-54. http://dx.doi.org/10.1097/SLA.0b013e318214b7e4

[2] S. Habarth, M. H. Samore, D. Lichtenberg and Y. Carmeli, "Prolonged Antibiotic Prophylaxis after Cardiovascular Surgery and Its Effects on Surgical Site Infections and Antimicrobial Resistance," Circulation, Vol. 101, No. 25, 2000, pp. 2916-2921.

http://dx.doi.org/10.1161/01.CIR.101.25.2916

[3] D. W. Bratzler, P. M. Houck, C. Richards, L. Steele, E. P. 
Dellinger, D. E. Fry, et al., "Use of Antimicrobial Prophylaxis for Major Surgery. Baseline Results from the National Surgical Prevention Project," Archives of Surgery, Vol. 140, No. 2, 2005, pp.174-182. http://dx.doi.org/10.1001/archsurg.140.2.174

[4] F. H. Edwards, R. M. Engelman, P. Houck, D. M. Shahian and C. R. Bridges, "The Society of Thoracic Surgeons Practice Guideline Series: Antibiotic Prophylaxis in Cardiac Surgery, Part I: Duration," The Annals of Thoracic Surgery, Vol. 81, No. 1, 2006, pp. 397-404. http://dx.doi.org/10.1016/j.athoracsur.2005.06.034

[5] F. Roques, S. A. Nashef, P. Micheal, E. Gauducheau, C. de Vincentis, E. Baudet, et al., "Risks Factors and Outcome in European Cardiac Surgery: Analysis of the EuroSCORE Multinational Database of 19030 Patients," European Journal of Cardio-Thoracic Surgery, Vol. 15, No. 6, 1999, pp .816-822. http://dx.doi.org/10.1016/S1010-7940(99)00106-2

[6] J. S. Garner, W. R. Jarvis, T. G. Emori, T. C. Horan and J. M. Hughes, "CDC Definitions for Nosocomial Infections," In: R. N. Olmsted, Ed., APIC Infection Control and Applied Epidemiology: Principles and Practice, Mosby, St Louis, 1996, pp. A1-A20.

[7] Antibioprophylaxie en Chirurgie et Médecine Interventionnelle. Société Française D'Anesthésie et de Réanimation, 2010.

http://www.sfar.org/article/669/antibioprophylaxie-en-chi rurgie-et-medecine-interventionnelle-patients-adultes-cc2010

[8] J. E. Conte, S. N. Cohen, B. B. Roe and R. M. Elashoff,
"Antibiotic Prophylaxis and Cardiac Surgery. A Prospective Double-Blind Comparison of Single-Dose versus Multiple-Dose Regimens," Annals of Internal Medicine, Vol. 76, No. 6, 1972, pp. 943-949. http://dx.doi.org/10.7326/0003-4819-76-6-943

[9] E. Tamayo E. J. Gualis, S. Florez, J. Castrodeza, J. M. Eiros Bouza and J. Alvarez, "Comparative Study of Single-Dose and 24-Hour Multiple-Dose Antibiotic Prophylaxis for Cardiac Surgery," The Journal of Thoracic and Cardiovascular Surgery, Vol. 136, No. 6, 2008, pp. $1522-$ 1527. http://dx.doi.org/10.1016/j.jtcvs.2008.05.013

[10] A. Konvalinka, L. Errett and I. W. Fong, "Impact of Treating Staphylococcus aureus Nasal Carriers on Wound Infection in Cardiac Surgery," Journal of Hospital Infection, Vol. 64, No. 2, 2006, pp. 162-168. http://dx.doi.org/10.1016/j.jhin.2006.06.010

[11] E. Bennett-Guerrero, T. B. Fergusson, M. Lin, et al., for the SWIPE-1 Trial Group, "Effect of an Implantable Gentamicin-Collagen Sponge on Sternal Wound Infection Following Cardiac Surgery. A Randomized Trial," The Journal of the American Medical Association, Vol. 304, No. 7, 2010, pp. 755-762.

http://dx.doi.org/10.1001/jama.2010.1152

[12] S. Stadler and T. Fleck, "Triclosan-Coated Sutures for the Reduction of Sternal Wound Infections? A Retrospective Observational Analysis," Interactive Cardiovascular and Thoracic Surgery, Vol. 13, No. 3, 2011, pp. 296-299. http://dx.doi.org/10.1510/icvts.2011.269001 